\title{
AHP FOR STUDENT DECISIONS IN A MONTESSORI ELEMENTARY CLASS
}

\begin{abstract}
In this paper we devise and use a new pairwise comparison questionnaire based upon a Liskert scale that enables Montessori elementary students to express their preferences for classroom jobs and areas of cleaning responsibility. In addition we develop the SimpleAHP web application so that elementary students can analyze the results of their questionnaires on their own. We find that the simplified questionnaire works well with our students and holds promise to allow more people access to AHP.
\end{abstract}

\section{Introduction}

Maria Montessori developed a teaching method in the early 1900's that is still popular today (Montessori, 2013). Part of that method is that students are given real responsibilities in their education, such as handling class rule making, discipline issues, and maintaining their classroom. In our class students are assigned areas of the classroom to clean, and jobs in those areas.

If we know students preferences for jobs and areas, we could try to assign them jobs they enjoy in areas they prefer, which could make them happier and more productive. We use a pairwise comparison process to accomplish this goal. A difficulty in this approach is the traditional 1-9 scale is hard to understand, especially for students aged 6-12. We decided to simplify the standard scale to a Likert type scale we call the EBM scale (meaning Equals, Better, and Much Better) and develop the SimpleAHP web application to perform the calculations and present the analysis in a way that students can navigate and understand. This research could help our class better handle job assignments, while the EBM scale approach and SimpleAHP web application could make AHP easier for anyone to understand.

\section{Literature Review}

It is difficult to find references for teaching youths to use AHP for decision making. In the paper (Liberatore, 1997) the authors explore using AHP in a higher educational setting, not a primary education setting. There were no tools available to help youth frame useful questions, solicit preference data, and analyze their results. We used the EBM scale, which is a Likert scale with 5 items, based upon the research in (Matell and Jacoby, 1971) for our simplified questionnaire format. We also created the SimpleAHP web application as a tool that youth could use to analyze their data, using the Shiny web toolkit (Chang et al., 2016) for the R (R Core Team, 2016) programming language.

\section{Hypotheses/Objectives}

Our research has three objectives: first to figure out the job and area preferences for our class, second to simplify the pairwise questionnaire process for ease of use for young students (although the simplification could be used by anyone), and third to create a tool students could use to analyze this simplified preference data. 


\section{Research Design/Methodology}

We decided to do a single goal AHP model, i.e. one pairwise comparison set, for the areas and another pairwise comparison set for the jobs (see Figure 1). We did this, in part, because leading 6-12 year olds through a complete comparison set for a full AHP model would be difficult. In addition, we wanted to create a process other students could follow to solve their own problems. A complex AHP/ANP model would be difficult for younger participants as well as those new to AHP/ANP.

We created a new questionnaire format using the EBM scale (see Figure 3 for an example), and decided upon default values for those votes of 1,4.5, and 9 respectively. We decided on the these numerical values for two reasons, first it breaks up the 1-9 scale into two equal segments, and secondly for our data it provided for good differentiation. Additionally we designed the SimpleAHP web application that allows for the use of the EBM scale and allows one to change their numerical values.

\section{Data/Model Analysis}

Our model for the jobs as well as the areas decision was a simple pairwise comparison among the alternatives. A visualization of the models, with a goal and alternatives can be found in Figure 1. The overall results by gender can be seen in Figure 2 and further results are available through the SimpleAHP web application at http: //tiny.cc/youthAHPOut1 for the areas and http://tiny.cc/youthAHPOut2 for the jobs.

Figure 1: Model Structure For Both Models

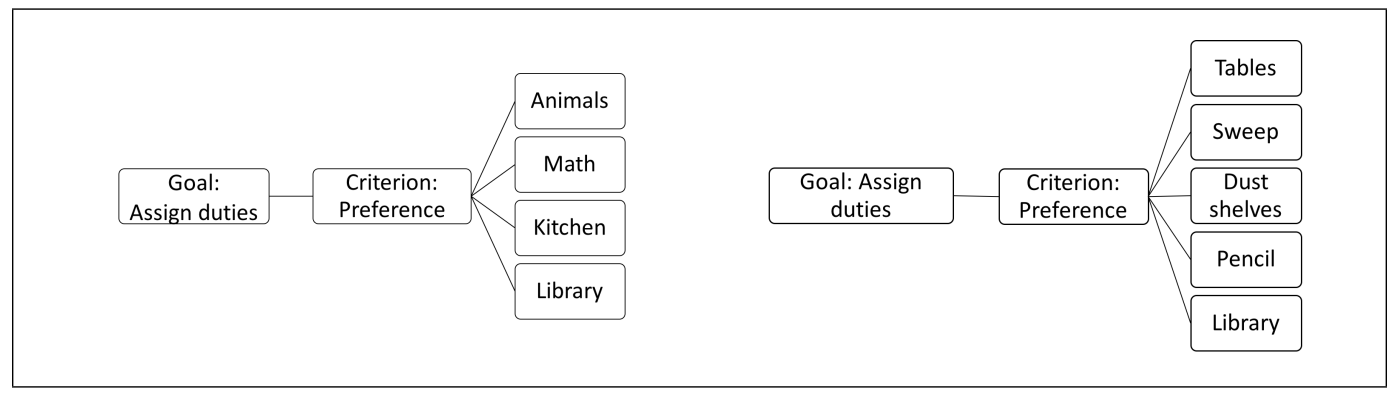

There was an interesting piece of work needed to reduce the number of job choices. The class actually has approximately 20 distinct jobs across the areas. Pairwise comparing 20 jobs is far too much for anyone to do. Therefore we searched for commonality across jobs to group like jobs together. After much work and discussion we were able to identify 5 job types, and that is what we used for the jobs choice question. We needed to make sure the job names indicated, intuitively, the meaning of the work being done, so that students would understand the pairwise comparison choices presented to them. 
Figure 2: Results by Gender
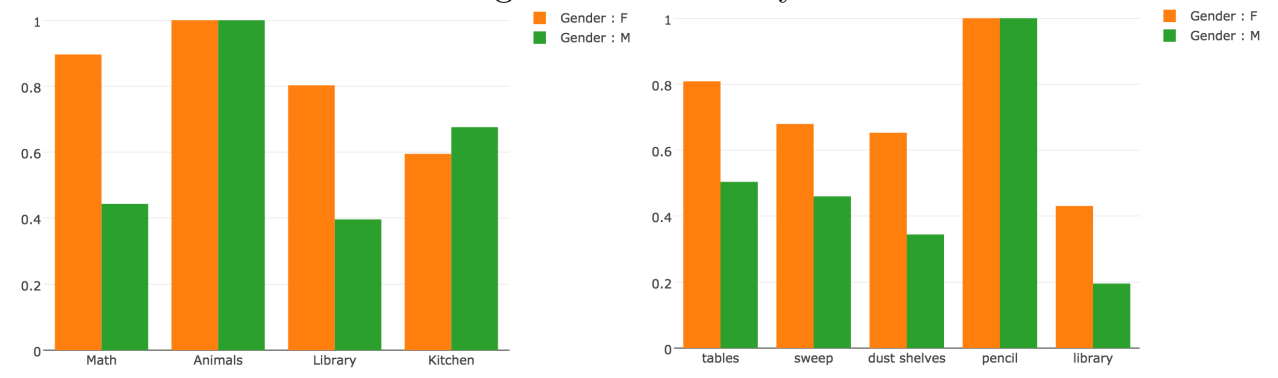

\section{Limitations}

In our particular models for jobs and areas, our pairwise votes are on the EBM scale, which is not as precise as the standard 1-9 scale. On the other hand, the standard 1-9 scale would have been more complicated than it should have been, especially for untrained students to participate in. (Our SimpleAHP application can accept votes either in the 1-9 scale or the EBM scale equally easily, so that limitation is not a problem for our application.)

In addition, we did not attempt to do either a full AHP or ANP model with the students. We felt this was necessary for two reasons. First, we did not want the students to get bogged down in too many pairwise comparisons. Second, we wanted to create a reproducible process that other students could follow. We were able to easily state our problems in this fashion, but other student decisions may not be amenable to this infrastructure.

\section{Conclusions}

The EBM scale, combined with the simplified questionnaire shown in Figure 3, allowed our young voters to easily participate and understand both the questions and the problems. The SimpleAHP web application allowed us to analyze our findings overall, by individuals, and by groups easily. In addition our process shows promise to be useful for other people who want to answer similar questions, without the need to understand all of the technical details of AHP/ANP theory.

\section{Key References}

Chang, W., Cheng, J., Allaire, J., Xie, Y., and McPherson, J. (2016). shiny: Web Application Framework for $R$. R package version 0.13.2.

Liberatore, Matthew J.and Nydick, R. L. (1997). Group decision making in higher education using the analytic hierarchy process. Research in Higher Education, $38(5): 593-614$.

Matell, M. S. and Jacoby, J. (1971). Is there an optimal number of alternatives for likert scale items? study. Educational and psychological measurement, 31:657-674.

Montessori, M. (2013). The montessori method. Transaction Publishers. 
R Core Team (2016). R: A Language and Environment for Statistical Computing. R Foundation for Statistical Computing, Vienna, Austria.

\section{Appendix 1}

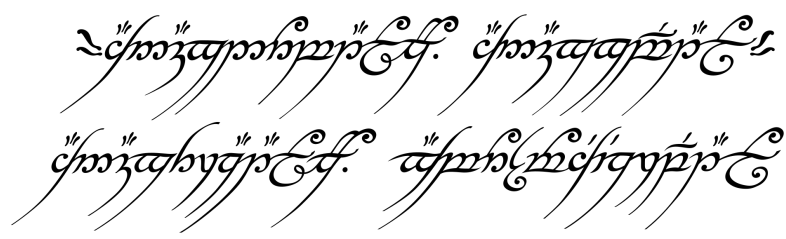

One ring to rule them all One ring to find them One ring to bring them all And in the darkness bind them

\section{Appendix 2}

Figure 3: Actual Questionnaire Students Filled Out For Areas Decision

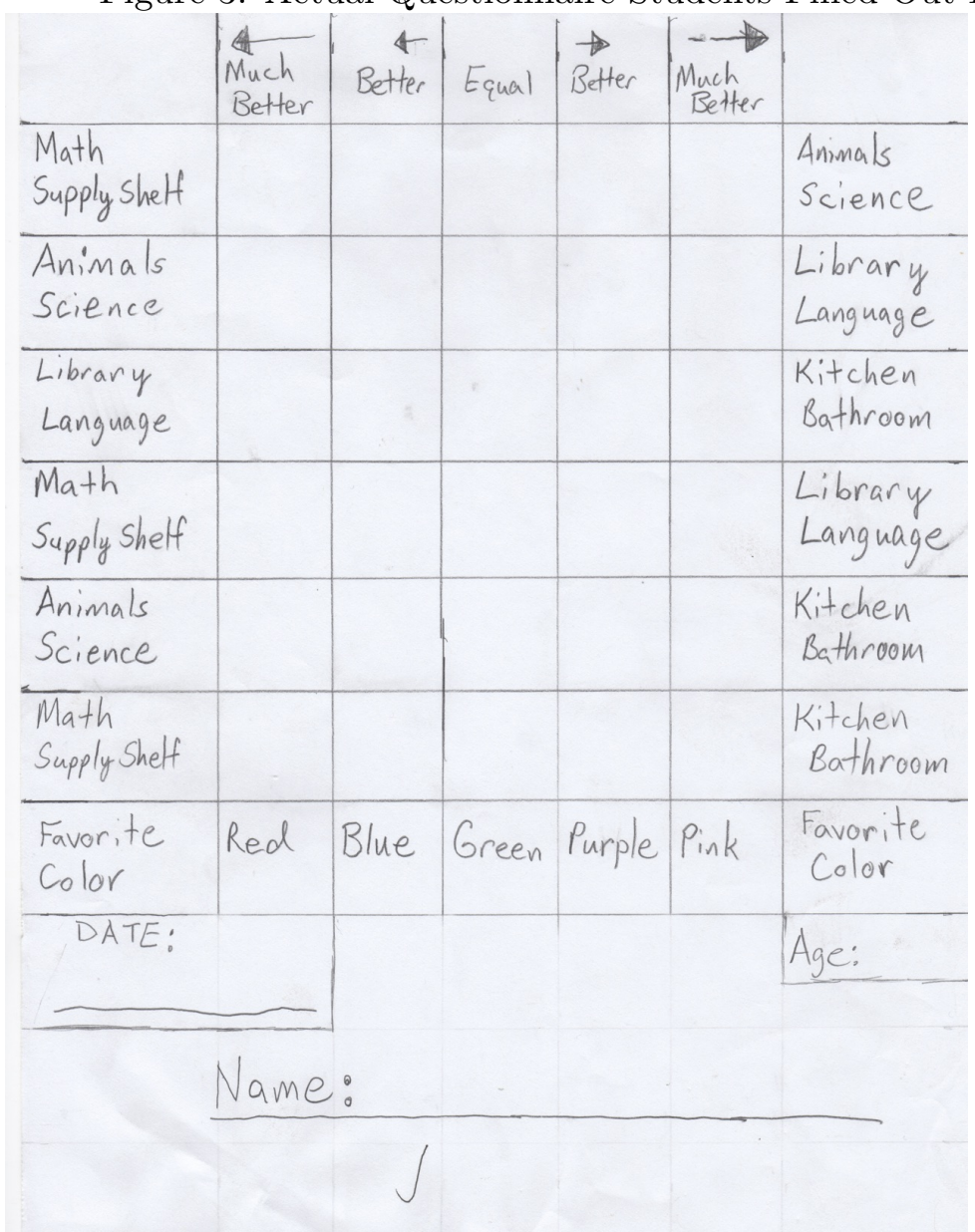

